\title{
Síndrome de Posner-Schlossman Relato de três casos
}

\section{Posner-Schlossman syndrome. Report on three cases}

Cesar Gomes da Silveira1', Paulo Ricardo Pereira de Oliveira², Renato Macedo da Silveira ${ }^{3}$

\section{ResUMo}

A Síndrome de Posner-Schollsman caracteriza-se por episódios intermitentes de glaucoma agudo acompanhados por leves uveítes anteriores. As crises são unilaterais, recorrentes e caracterizadas por pupilas discretamente dilatadas, ao contrário, das pupilas mióticas, mais comumente vistas nas irites. Neste artigo temos o relato de três casos de pacientes do sexo masculino com síndrome de Posner-Schlossman (SPS), possuindo dois deles um grau de consanguinidade em primeiro grau. Baseando-se em sinais e sintomas típicos, e cuidadoso exame oftalmológico, que aqui são discutidos e analisados, é possível realizar o diagnóstico da crise glaucomatociclítica. A rara ocorrência da doença em questão justifica a importância de um diagnóstico preciso para seu tratamento correto a fim de evitar cirurgias desnecessárias ou doenças secundárias por equívocos diagnósticos.

Descritores: Uveíte/diagnostico; Pressão intraocular; Síndrome; Glaucoma/diagnóstico

\footnotetext{
${ }^{1}$ Médico Residente do primeiro ano do Centro de Estudos e Pesquisas Oculistas Associados - CEPOA - Rio de Janeiro ( RJ), Brasil; ${ }^{2}$ Médico Oftalmologista e Professor Assistente da Faculdade de Medicina da Universidade Luterana do Brasil - ULBRA - Canoas (RS), Brasil;

${ }^{3}$ Médico Oftalmologista. Chefe e presidente do Centro de Estudos da Clínica de Olhos Santa Lúcia, Cruz Alta (RS), Brasil. 


\section{INTRODUÇÃO}

$\mathbf{E}$ m 1948, Posner e Schlossman relataram o primeiro caso concreto de crise glaucomatociclítica, descrevendo assim uma síndrome rara e de importante distinção entre os tipos de glaucoma associados a uveítes. ${ }^{(1,2)}$

A maioria dos autores considera a sua etiologia desconhecida, ${ }^{(1,3-8)}$ entretanto uma série de trabalhos mais recentes estuda possíveis relações de outros agentes, como o herpes simplex, o citomegalovírus, e o helicobacter pylori, ${ }^{3,9-11)}$ bem como a associação com HLA-Bw54 positivo em $40 \%$ dos pacientes com síndrome de PosnerSchlossman (SPS). ${ }^{(4,6)}$ Contudo, ainda não se pode atribuir definições absolutas em relação à causa da doença.

Em um dos estudos, ${ }^{(9)}$ pesquisando associação com o virus Herpes Simplex (HSV), observou-se a presença de fragmentos de DNA do HSV em amostras de humor aquoso dos pacientes com SPS, enquanto que no grupo controle o resultado foi negativo na pesquisa do vírus, sugerindo a possibilidade de associação do HSV com a síndrome. . $^{(3,9)}$

Relatos recentes indicam que não há associação de citomegalovírus (CMV) como causa da crise glaucomatociclítica. ${ }^{(10)}$

Ainda em investigação de possíveis causas, outro estudo $^{(11)}$ realizou uma análise sorológica para pesquisa de Helicobacter Pylori em associação ou não com SPS. ${ }^{(11)}$ Também houve divisão entre pacientes com SPS e grupo controle. Foram encontrados, em $80 \%$ do grupo com SPS, os anticorpos anti-IgG, para H. Pylori no soro, enquanto que no grupo controle a taxa de positividade foi de $56,2 \%$, estando o H. Pylori relatado como um importante fator de risco para SPS. ${ }^{(11)}$

Ainda outros mecanismos têm sido postulados: possíveis processos vasculares anormais e/ou mecanismos imunológicos, aumento dos níveis de prostaglandinas, defeitos anatômicos e condições alérgicas, hipersecreção aquosa, e reação mediada por $\operatorname{IgE} .^{(4,12)}$

A SPS é mais reconhecida em adultos jovens, do sexo masculino entre 20 e 50 anos de idade..$^{(1-7)}$ Porém há relatos de sua ocorrência também em crianças, e mais raramente em idosos. ${ }^{(1,2)}$

As crises se caracterizam pela ocorrência de ataques agudos e recorrentes de uveíte anterior leve, associado ao aumento da pressão intraocular (PIO) - entre 40 e $60 \mathrm{~mm} \mathrm{Hg}$ - unilateralmente, quase sempre. Entretanto em uma porcentagem dos casos há envolvimento bilateral não-simultâneo. (1-8,12) $^{(12}$

Cada crise pode se estender de poucas horas a várias semanas, mas em geral cessam entre 7 e 14 dias. A frequência dos ataques varia, com períodos de remissão que podem durar de um mês a muitos anos, de modo que com o passar do tempo o intervalo entre as crises tende a ser maior. ${ }^{(1-5)}$
Os sintomas mais frequentes são leve desconforto ocular, diminuição da visão e aparecimento de halos coloridos em torno de luzes. ${ }^{(1-3,7,8)}$

À inspeção, observa-se um olho calmo com discreta hiperemia conjuntival. ${ }^{(1-3,7)}$

Cabe salientar que a literatura atual também considera importante o acompanhamento efetivo de pacientes com síndrome de Posner-Schlossman, pois um percentual significativo desses poderá desenvolver glaucoma crônico de ângulo aberto, o que sugere assim, uma alteração degenerativa da malha trabecular, dificultando o escoamento do humor aquoso. ${ }^{(1,2,4-6)}$

Sugere-se que o diagnóstico da crise glaucomatociclítica deva se dar através de um rigoroso acompanhamento do caso, sendo identificadas as repetidas crises de ciclíte, geralmente autolimitadas, somadas a um quadro clínico característico. ${ }^{(5)}$

Ao diagnóstico diferencial, deve-se considerar glaucoma primário de ângulo estreito com bloqueio pupilar ou outras uveítes hipertensivas, principalmente a ciclíte heterocrômica de Fuchs, na qual o glaucoma tende a se desenvolver mais tardiamente, pois o aumento da PIO se dá pela deposição de células inflamatórias na malha trabecular e à esclerose desta. ${ }^{(1,5)}$

O tratamento visa a minimizar os danos causados pela pressão sobre o nervo óptico, ao longo do tempo, e reduzir a inflamação. ${ }^{(7,12)}$

É importante já se destacar que, a longo prazo, os corticóides poderão causar a elevação da PIO induzindo riscos adicionais de glaucoma secundário e/ou opacificação subcortical posterior do cristalino i.e. catarata secundária. ${ }^{(1,5)}$

Atualmente existem relatos do uso experimental de tonômetro portátil (Phosphene), cujo mecanismo possibilita a automonitorização e controle da PIO pelo paciente em uso domiciliar, nos períodos de remissão dos ataques. $^{(13)}$

Classicamente a cirurgia pode não estar indicada na SPS; porém, em alguns casos, com recorrência elevada, poderá se instalar glaucoma crônico simples de ângulo aberto, levando a necessidade de manutenção cirurgica. ${ }^{(1,5,7,8)}$

Os autores consideram importante este relato de caso por sua raridade e pela importância do diagnóstico preciso para seu tratamento correto, evitando, deste modo, terapêutica clínica imprópria e/ou cirurgias desnecessárias. ${ }^{(1,5)}$

\section{Descrição dos casos e resultados}

\section{Caso 1}

F.G.B., 44 anos, sexo masculino, branco, casado, agrônomo, procedente do interior do Estado do Rio Grande do Sul.

Em 1998 procurou atendimento oftamológico com 
relato de várias crises de "uveíte hipertensiva" em olho direito (OD), há 28 anos, com "pesquisas clínicas e laboratoriais negativas para classificação da uveíte, e acompanhamento oftalmológico prévio". Relatou ainda glaucoma em avô e tio (sic). Nega trauma ocular e infecção em atividade. Referiu tosse seca após início do uso de colírios.

Estava em uso diário de acetazolamida $250 \mathrm{mg}$, por via oral (VO), latanoprost $50 \mathrm{mcg} 1 \mathrm{x} / \mathrm{dia}$, maleato de timolol $0,5 \% 1 \mathrm{x} /$ dia, e acetato de prednisolona a $1 \%$ de 4/4 horas, tópicos.

Ao exame ocular apresentou edema de córnea, íris heterocrômica, leve midríase, flare raro e precipitados retroceráticos $(\mathrm{PK})$ no terço inferior da córnea, com opacificação cristaliniana subcortical posterior inicial em OD, e fundoscopia normal (Figura 1). O olho esquerdo (OE) não apresentava alterações dignas de nota.

$\mathrm{O}$ exame refracional demonstrava astigmatismo misto em OD e emetropia em olho esquerdo (OE), atingindo $20 / 20$.

A PIO do OD era de $16 \mathrm{~mm} \mathrm{Hg}$ e a PIO do OE 12 mm Hg (10:30 h), por tonometria de aplanação de Goldmann. A gonioscopia apresentava ângulo aberto em $360^{\circ}$.

Com estes dados, foi realizado o diagnóstico de SPS e instituído o tratamento, com a manutenção de latanoprost $50 \mathrm{mcg}$, às 21 horas, cloridrato de dorzolamida tópico de 12/12 horas, e cetorolaco de trometamina, $0,4 \% 5 \mathrm{x} / \mathrm{dia}$, e tropicamida $1 \% 3 \mathrm{x} / \mathrm{dia} .^{(1,-4,12)}$ Após dez dias, em revisão, o OD apresentava ausência de flare, PK e PIO de $10 \mathrm{~mm}$ $\mathrm{Hg}$, tendo sido então sugerida a suspensão do tratamento, com controle ambulatorial subsequente.

Quatro meses depois, o paciente recorre com queixas de OD dolorido e hiperemiado há dois dias. Apresentava PIO em OD de $28 \mathrm{~mm} \mathrm{Hg}$ (14:00 h), PK finos, e flare $++++/ 4+$. OE sem alterações. Foi reinstituído o mesmo tratamento da crise anterior, com revisões semanais. Na primeira semana de acompanhamento, a PIO em OD se estabeleceu em $10 \mathrm{~mm} \mathrm{Hg}$ (14:00 h), os aspectos inflamatórios desaparecem, mas com PK finos ainda presentes. Optou-se pela redução gradual da medicação, mas com a suspensão do inibidor da anidrase carbônica. Na semana seguinte os padrões oculares se mantiveram em ambos os olhos (AO), porém com redução nos PK para +/4+. Destituiu-se o tratamento.

O paciente permaneceu por um ano e meio sem crises, quando em 2000 tornou a apresentar nova crise. O tratamento instituído foi idêntico ao da primeira crise, somando-se o uso de acetazolamida, $500 \mathrm{mg} / \mathrm{dia}$, VO. ${ }^{(1,4-8,12)}$ A evolução foi favorável, sendo retirados, gradualmente, os medicamentos.

Após isso, passou a apresentar 2 a 3 episódios por ano de crise glaucomatociclítica em OD. Comparecia às consultas durante as crises, com queixas de hiperemia ocular, visão embaçada, halo nas luzes, desconforto ocu-

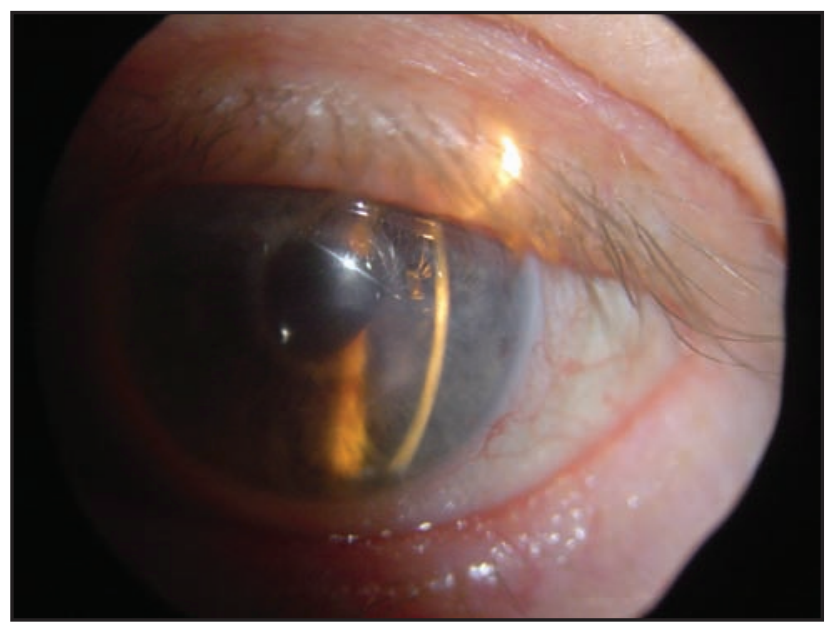

Figura 1: Olho direito : discreta atrofia de íris e edema de córnea devido ao aumento da pressão intra-ocular; Fotografia do paciente em crise. Cortesia dos arquivos da Clínica de Olhos Santa Lucia - Cruz Alta - RS

lar (sic). Chegou a apresentar, em algumas crises, níveis pressóricos de $44 \mathrm{~mm} \mathrm{Hg}$ em OD, enquanto no OE, manteve-se assintomático e com PIO média de $10 \mathrm{~mm} \mathrm{Hg}$.

Quando instalada a crise, o tratamento com AINE, cicloplégico leve e hipotensor ocular demonstrou sempre ser efetivo.

Foram realizados exames de campo visual computadorizado que se mantiveram normais no decorrer da evolução do paciente.

Em abril de 2007, o paciente procurou atendimento com OE normal, apresentando PIO de $10 \mathrm{~mm} \mathrm{Hg}$ (11:00 h) e acuidade visual (AV) de 20/20, enquanto OD apresentava hiperemia bulbar +++/4+, Fundus Ocular impossível, PK +++/4+, edema de córnea +++/4+, PIO de $43 \mathrm{~mm} \mathrm{Hg}$ (11:00 h) e AV de 20/200. Na ocasião, foi instituído novamente o tratamento com latanoprost $50 \mathrm{mcg}$, às 21 horas, cloridrato de dorzolamida tópico de 12/12 horas, e cetorolaco de trometamina, $0,4 \% 5 \mathrm{x} / \mathrm{dia}^{(1,4-8,12)}$ e tropicamida $0,1 \% 3 \mathrm{x} /$ dia, porém sem remissão de sintomas.

Sugeriu-se a infiltração subconjuntival de corticóide, mas após acompanhamento seriado por duas semanas, não se observou baixa da PIO no OD, aquém da média de $38 \mathrm{~mm} \mathrm{Hg}$.

Foi sugerida cirurgia filtrante, considerando-se a evolução de todas as crises, objetivando a manutenção da visão do paciente.

O procedimento de eleição foi a trabeculectomia, sendo administrado o seguinte tratamento no pós-operatório: cloridrato de moxifloxacino $0,5 \% 5 \mathrm{x} / \mathrm{dia}$, cicloplégico colírio $3 \mathrm{x} /$ dia e acetato de prednisolona $1,0 \% 4 \mathrm{x} / \mathrm{dia} .{ }^{(14)}$

Na primeira semana após a cirurgia, o OD apresentou-se com câmara anterior preservada, sem sinais inflamatórios, edema de córnea discreto e PIO de 16 $\mathrm{mm} \mathrm{Hg}(10: 30$ h). O antiinflamatório esteróide foi então 
substituído por AINE tópico (diclofenaco sódico 0,1\%, $3 x /$ dia), embora haja a sugestão para a manutenção desse medicamento por tempo mais prolongado.

Manteve-se acompanhamento sequencial semanal, e o pós-operatório de 30 dias apresentou olho sem sinais inflamatórios, edema de córnea no terço inferior leve e AV corrigida para 20/25. A conduta sugerida manteve o AINE colírio, sendo gradualmente retirado.

Em novembro de 2007, o paciente retornou à consulta com queixa de "névoa" em OD. Ao exame apresentava discreta irite, PIO do OD de $12 \mathrm{~mm} \mathrm{Hg}$ (10:00 h) e PIO do OE de $10 \mathrm{~mm} \mathrm{Hg}(10: 00 \mathrm{~h})$. Foi prescrito novamente o mesmo AINE em doses decrescentes.

Em sua última consulta, em fevereiro de 2008, apresentou ambos os olhos dentro da normalidade, com PIO OD de $13 \mathrm{~mm} \mathrm{Hg}$ e PIO OE de $11 \mathrm{~mm} \mathrm{Hg}$, sem uso de medicação.

\section{Caso 2}

A.D.B., 22 anos, sexo masculino, branco, solteiro, estudante, procedente de Santo Ângelo-RS.

No início de 2007, paciente procurou atendimento para saber se possuía o mesmo diagnóstico que o seu pai (Caso 1). Estava acompanhando com oftalmologista de sua cidade. Referiu seis crises de aumento da PIO em olho esquerdo (sic), com a última medida de $42 \mathrm{mmHg}$ em OE, com OD normal na ocasião da consulta anterior (sic). Usou na última crise, que se encerrou há alguns dias da data desta consulta, dexametasona $0,1 \%$ tópico, atropina tópica $0,5 \% 3 \mathrm{x} /$ dia, maleato de timolol 0,25\% tópico, $2 \mathrm{x} / \mathrm{dia}$, sob indicação do médico de origem.

Possui história familiar de síndrome de PosnerSchlossman confirmada em genitor do sexo masculino (Caso 1).

Ao exame inicial, apresentou ambos os olhos sem sinais inflamatórios, e segmento anterior normal, à exceção de anisocoria discreta, com midríase média reagente em OE, e OD sem alterações.

À fundoscopia, foi observada escavação papilar grau I/IV em OD e grau II/IV em OE. Não apresentava ametropias dignas de nota.

No exame de biomicroscopia não apresentou alterações da normalidade. Apresentava-se com PIO de OD $14 \mathrm{~mm} \mathrm{Hg}$ e PIO de OE de $20 \mathrm{mmHg}$ (15:00 h), por tonometria de aplanação de Goldmann e gonioscopia normal.

Considerando como hipótese plausível a SPS, paciente recebeu colírio de maleato de timolol 0,5\%, 2x/ dia por um mês, e suspensão do colírio de corticoesteróide. Um mês depois, retornou ao exame apresentando PIO de OD de $14 \mathrm{~mm} \mathrm{Hg}$ e PIO de OE de $12 \mathrm{~mm} \mathrm{Hg}$. Realizou-se campo visual computadorizado na ocasião, em $\mathrm{AO}$, não apresentando alterações campimétricas significativas.

Sugeriu-se reencaminhamento ao seu oftalmolo- gista original, com sugestão diagnóstica de SPS em OE, e com orientação para abstenção do uso de corticoesteróide tópico crônico, para se evitarem complicações decorrentes de seu uso a longo prazo.

\section{Caso 3}

P.R., 43, masculino, branco, casado, comerciante, procedente de Ibirubá - RS.

Em 2001, paciente procurou atendimento na C.O.S.L de Cruz Alta - RS relatando "glaucoma em OD, há 10 anos", tendo passado por várias avaliações oftalmológicas anteriores (sic), com crises intermitentes de dor e perda de visão(sic), e "enxergando arcos de luz".

Informou o uso de colírios de acetato de fluormetolona $0,001 \mathrm{~g}$, acetato de prednisolona $1,2 \mathrm{mg} /$ $\mathrm{ml}$, pilocarpina, maleato de timolol e cloridrato de dorzolamida associados, $2 \mathrm{x} /$ dia. Na primeira consulta estava em uso de AINE tópico, 3x/dia.

Ao exame, hiperemia bulbar em OD e anisocoria com leve midríase à direita. Ao exame de fundo de olho, encontrou-se escavação de papila grau II/IV em OD, com OE preservado.

A biomicroscopia revelava flare escasso, e PK no terço inferior da córnea com opacificação cristaliniana subcortical posterior central em OD. Gonioscopia sem alterações.

A AV aferida foi 20/80 e J6 em OD.O olho esquerdo, emétrope.

A PIO do OD era de $36 \mathrm{~mm} \mathrm{Hg}$ e $10 \mathrm{~mm} \mathrm{Hg}$ a PIO do OE (11:30h), à tonometria de aplanação de Goldmann.

Baseando-se nesses achados, cogitou-se possível SPS e se iniciou o tratamento tópico com tropicamida $1 \%$, tartarato de brimonidina $0,15 \%$, maleato de timolol e cloridrato de dorzolamida em associação $2 \mathrm{x} / \mathrm{dia}$.

Uma semana depois, em nova reavaliação, apresentou AV de 20/30 em OD. Persistia o flare mínimo e PIO do OD era de $12 \mathrm{~mm} \mathrm{Hg}$ (11:00 hrs). Foi acrescentado colírio de cetorolaco de trometamina $0,5 \%, 2 \mathrm{x} / \mathrm{dia}$, mantendo-se os demais colírios por quatro semanas restantes.

Um mês depois, sua AV sem correção no OD foi de 20/25 e J3. PIO do OD $8 \mathrm{~mm} \mathrm{Hg}$, com ausência de flare. Recebeu alta, com manutenção do colírio de tropicamida $1 \%, 1 \mathrm{x} /$ dia, por um mês.

Cinco meses depois, paciente retornou com queixa de dor e "sensação de pressão no olho", há dois dias. A AV sem correção: OD 20/40 e J5 / OE 20/25 e J1. A PIO média do OD foi de $26 \mathrm{~mm} \mathrm{Hg}$ e PIO do OE $10 \mathrm{~mm} \mathrm{Hg}$. Apresentava um flare importante, $+++/ 4$. Novamente, foi instituído cetorolaco de trometamina $0,5 \%, 2 \mathrm{x} / \mathrm{dia}$, tropicamida $1 \%, 3 x /$ dia, e maleato de timolol associado a cloridrato de dorzolamida, $2 \mathrm{x} /$ dia. Uma semana depois, à biomicroscopia, apresentava flare raro, PIO de 
OD $12 \mathrm{~mm} \mathrm{Hg}$. Manteve-se a tropicamida 1\% 3x/dia, por mais duas semanas, acrescido de AINE colírio $3 \mathrm{x} /$ dia por uma semana.

Em nova revisão, não apresentava alterações oculares, com PIO média de $8 \mathrm{~mm} \mathrm{Hg}$ em ambos os olhos.

Após três meses, retornou à consulta com queixas de dor "em pressão" em OD, há cerca de 24 horas. Sua AV era de 20/40 e J3 em OD. À biomicroscopia, flare importante novamente, com PIO de OD de $38 \mathrm{~mm} \mathrm{Hg}$ e PIO de OE $10 \mathrm{~mm} \mathrm{Hg}$ (14:00 h). Diante de outra crise, optou-se por AINE colírio (flurbiprofeno 0,03\%) 5x/dia, tropicamida $1 \% 3 x /$ dia, maleato de timolol associado a cloridrato de dorzolamida, $2 \mathrm{x} / \mathrm{dia}$, e tartarato de brimonidina $0,15 \%, 2 \mathrm{x} /$ dia.

Dez dias depois, o paciente se mostrava assintomático, sendo então, destituído o tratamento.

Mais uma vez, após dez meses apresentou novo episódio similar aos anteriores: com PK e flare brando em OD. PIO de OD 36 mm Hg e PIO de OE 12 mm Hg. Repetiu-se a terapia da última crise, por uma semana.

Na revisão, após uma semana, estava com PIO de OD ainda elevada (22 mmHg). Seguiu-se, então, com acompanhamento semanal, e com o tratamento mantido. Houve, entretanto, um aumento da PIO em OD para $30 \mathrm{~mm} \mathrm{Hg}$, em uma semana após a consulta anterior. A partir disso, foi associado o uso de colírio de prostaglandina (travaprosta $0,004 \%$ ) $1 x$ à noite. Após uma semana, o paciente apresentou PIO do OD de 10 $\mathrm{mm} \mathrm{Hg}$, o olho calmo, e assintomático.

Passados dois meses, apresentou novo episódio com remissão do quadro em uma semana, em resposta à última terapêutica proposta.

Manteve-se estável, ao passar de um ano assintomático e sem novas recorrências de SPS.

Em outubro de 2004, teve novo episódio da SPS, com PIO de OD chegando à média de $38 \mathrm{~mm} \mathrm{Hg}$, e 12 $\mathrm{mm} \mathrm{Hg}$ no OE. Teve remissão dos sintomas em duas semanas de tratamento. Permaneceu, então até o ano de 2007, assintomático.

A última crise glaucomatociclítica ocorreu em outubro de 2007 com o quadro sintomático idêntico ao da primeira consulta. PIO do OD de $30 \mathrm{~mm} \mathrm{Hg}$ e $8 \mathrm{~mm}$ $\mathrm{Hg}$ em OE. Usou colírios de brimonidina e maleato de timolol, cetorolaco de trometamina $0,5 \%$ e tropicamida $1 \%$, por uma semana, reapresentando olho "calmo" e PIO de OD $10 \mathrm{~mm} \mathrm{Hg}$.

Mantém-se desde outubro de 2007 estável, sem recorrência dos sintomas.

\section{Discussão}

Os casos relatados são uma combinação de sinais e sintomas típicos da síndrome de Posner-Schlossman, que se caracteriza por manifestações glaucomatosas e ciclíticas específicas. ${ }^{(18,12,15)}$ De todos os pacientes com diagnóstico de uveíte, 1.19 por cento apresentam esta síndrome. ${ }^{(1)}$ Quando se classifica glaucoma secundário à uveítes, a síndrome de Posner-Schlossman é responsável por apenas $0,9 \%$ dos casos. ${ }^{(16)}$

Caracterizada por acometimento unilateral, na maioria dos casos, ${ }^{(1,3-6)}$ e tipicamente em adultos masculinos na faixa etária de 20 a 50 anos,,$^{(1-7)}$ a doença evolui com episódios recorrentes de frequiência variada, como podemos ver aqui. ${ }^{(1-6)}$

Na crise, um desconforto ocular leve é o sintoma mais frequente, seguido de discreta diminuição da AV e aparecimento de halos coloridos. ${ }^{(1-3)}$ Nos casos expostos acima, encontramos tanto estes sintomas, como também os sinais de ciclíte revelados na biomicroscopia e o aumento significativo da PIO, chegando à máxima média de $44 \mathrm{~mm} \mathrm{Hg}$ no paciente do Caso 1, no período dos ataques.

Ainda, à biomicroscopia, observou-se midríase do olho afetado nos três casos relatados. Esse achado contraria a presença de miose que normalmente pode se fazer presente durante crises de irite, e não se formam sinéquias anteriores ou posteriores. . $^{(1-5,7)}$

Além disso, encontramos PK concentrados no terço inferior da córnea, íris heterocrômica, flare, edema de córnea e hiperemia bulbar, os quais são típicos da doença relatada. ${ }^{(1-7,15)}$ É importante destacar a ausência de sinéquias. ${ }^{(1-6)} \mathrm{A}$ gonioscopia mostrou o seio camerular aberto nos quatro quadrantes, em todos os casos. ${ }^{(1,4,5)}$

A PIO do olho contralateral manteve-se sempre dentro da normalidade, independente das crises. A PIO do olho afetado, fora dos períodos de crise glaucomatociclítica, apresentou-se também dentro de valores fisiológicos.

Os tratamentos instituídos se mostraram eficazes com uso de corticóide tópico e/ou AINE durante as crises para conter o processo inflamatório, ${ }^{(1,4-8)}$ associados a hipotensores oculares e cicloplégico, conforme demonstra a literatura. ${ }^{(6,8)}$

Porém, com a recorrência dos ataques o tratamento farmacológico mostrou-se ineficiente ao longo dos anos no paciente do Caso 1, instalando-se diminuição da AV, edema epitelial importante (+++/4+) e fundus ocular impossível. Esse quadro levou a realização da cirurgia filtrante, como sugere a bibliografia. ${ }^{(1,4,8)}$

Como diagnóstico diferencial devemos considerar o glaucoma agudo primário e de uma forma mais complexa, outras formas de uveíte associadas a glaucoma, principalmente a Ciclite Heterocrômica de Fuchs.

Aqui é importante mencionar que na síndrome de Posner-Schlossman a hipertensão intra-ocular ocorre em média de 1 a 2 dias antes da uveíte, podendo acontecer sem esta. ${ }^{(1)}$ No glaucoma secundário à uveíte, como a nomeação sugere, o glaucoma é uma complicação da 
Tabela 1

Características das apresentações dos casos de síndrome de Posner-Schlossman

\begin{tabular}{|c|c|c|c|}
\hline & Caso 1 & Caso 2 & Caso 3 \\
\hline Sexo & Masculino & Masculino & Masculino \\
\hline Idade & 44 anos & 22 anos & 43 anos \\
\hline HF de glaucoma & Sim & Sim & Sim \\
\hline \multirow[t]{2}{*}{ Queixas } & $\begin{array}{l}\text { Hiperemia bulbar } \\
\text { Visão embaçada }\end{array}$ & $\begin{array}{l}\text { Seis crise de aumento de PIOE } \\
\text { Perda de visão }\end{array}$ & Dor e pressão no olho \\
\hline & Halo nas luzes & & Arcos nas luzes \\
\hline Olho (s) acometido (s) & OD & $\mathrm{OE}$ & OD \\
\hline PIO Máxima* & $44 \mathrm{OD}$ & $20 \mathrm{OE}^{* * *}$ & $38 \mathrm{OD}$ \\
\hline Acometimento contralateral & - & - & - \\
\hline \multirow[t]{6}{*}{ Achados ** } & Íris heterocrômica, & Midriase média OE & Hiperemia bulbar, \\
\hline & Midríase leve OD & Olho calmo & Midríase leve OD \\
\hline & Flare raro & & Flare raro/importante \\
\hline & $\mathrm{PK}$ & & PK \\
\hline & Catarata inicial & & Catarata inicial \\
\hline & Edema de córnea & & Edema de córnea \\
\hline Fundo de olho & Normal & Escavação papilar: & Escavação papilar: \\
\hline & & OD grau I/IV, & OD grau II/IV, \\
\hline & & OE grau II/IV & OE normal \\
\hline \multirow[t]{2}{*}{ Complicações } & Catarata & - & - \\
\hline & Glaucoma de ângulo aberto & & \\
\hline Duração & 10 dias & 4 semanas & 7 a 21 dias \\
\hline Frequência & 2 a 3 crises por ano & - & $\begin{array}{l}3 \text { crises/ano } \\
\text { remissão por } 1 \text { ano } \\
2 \text { crises/ano } \\
\text { remissão por } 2 \text { anos } \\
1 \text { crise/ano } \\
\text { remissão por } 3 \text { anos }\end{array}$ \\
\hline Cirurgias & Trabeculectomia & - & - \\
\hline Evolução & Irites sem HIO & $\begin{array}{l}\text { Acompanha com } \\
\text { médico de origem }\end{array}$ & 2 anos assintomático \\
\hline
\end{tabular}

* Pressão medida em mmHg por tonometria de aplanação de Goldman.

** Achados referentes ao olho afetado. Presume-se olho contralateral normal.

*** Paciente vinha recebendo tratamento com hipotensores, porém referiu $42 \mathrm{mmHg}$ no olho acometido.

Legenda: HF de glaucoma: Historia familiar de glaucoma. PIOE: Pressão intra-ocular do olho esquerdo. PIO máxima: Pressão intraocular máxima. OD: Olho direito. OE: Olho esquerdo. PK: Precipitados retro-ceráticos. HIO: hipertensão intra-ocular.

uveíte instalada e a clínica, então, difere: identifica-se a presença de sinéquias, miose e pupila irregular. A câmara anterior apresenta sinais inflamatórios caracteristicamente importantes e os PK variam em maior número na maioria dos casos, podendo ser pigmentados. ${ }^{(1,15)}$

Os casos relatados trazem todas as características da síndrome de Posner-Schlossman, contudo cabe salientar que, no Caso 2, não ocorreu um pico hipertensivo intraocular marcadamente expressivo como nos demais, pois o paciente já se mantinha em tratamento no momento da consulta.

No Caso 3, observa-se uma freqüência aparentemente maior de recidivas e também uma duração em seus episódios, em média, superior aos outros e à descrita na literatura, a qual sugere que cada crise cesse entre 7 e 14 dias. ${ }^{(1-5)}$ Ainda se observou diminuição na freqüência das crises com o passar do tempo, com maiores períodos de remissão, concordando com a literatura. ${ }^{(1-5)}$
Cabe por último salientar que embora existam algumas considerações quanto ao uso de análogos de prostaglandinas em glaucoma inflamatório, lançamos mão dessa classe de droga para auxiliar a manutenção da PIO elevada e de difícil controle, uma vez que não há contra-indicação absoluta ao seu uso, e nos casos descritos obteve-se êxito com seu uso, considerando que é de suma importância atingir e manter a PIO em valores normais. Na tabela 1 é possível observar as diferenças entre os três casos.

\section{ConClusão}

Os pacientes descritos apresentaram quadros clínicos bem característicos de síndrome de PosnerSchlossman, permitindo sua exemplificação fidedigna.

Os pacientes dos Casos 1 e 2 possuem laços de consangüinidade de primeiro grau. Ainda assim, o genitor 
paterno (Caso 1) referiu antecedentes de glaucoma em tio e avô o que, sugere a possibilidade de uma SPS não diagnosticada no passado. A presença de SPS em seu filho (Caso 2) também nos sugere a possibilidade de linearidade genética.

A SPS parece ser inicialmente autolimitada, porém, com o tempo, pode resultar em glaucoma crônico de ângulo aberto e em conseqüente perda permanente da AV. Portanto, a importância do diagnóstico correto reflete no estabelecimento do tratamento efetivo.

Contudo o fato de ser uma patologia rara, autolimitada e de etiologia incerta, dificulta a elaboração de um estudo de acompanhamento dos efeitos das drogas na doença. ${ }^{(17)}$

Assim nos vale o cuidado imperativo com o uso de glicocorticóides tópicos na síndrome, sendo eles responsáveis por possíveis iatrogenias, como as descritas anteriormente-glaucoma e catarata.

Os pacientes com diganóstico de SPS podem ser tratados com colírios hipotensores, somados a um cicloplégico leve e AINE no período das crises. Embora seja difícil estabelecer um padrão terapêutico devido à raridade da doença, esses esquemas acima retratados são suficientes, como podemos ver. Guarda-se o procedimento cirúrgico para casos seletos e refratários ao tratamento proposto.

\section{Abstract}

Posner-Scholssman Syndrom is characterized by intermittent acute episodes of glaucoma with mild anterior uveitis. Usually, the crisis is unilateral, recurrent and typified by discreet dilation of the pupils as opposed to constricted pupils, commonly seen in iritis. Herein this article, we report three cases of male patients with PosnerSchlossman syndrome, two of them showing a significant degree of first degree parenthood. Based upon typical signs and symptoms, and careful eye examination, thereby discussed and analyzed, it is possible to diagnose the glaucomatocyclitic crisis. The rare occurrence of the disease justifies the importance of an accurate diagnosis for a proper management in order to avoid unnecessary surgery or secondary diseases by diagnostic mistakes.

Keywords: Uveitis/diagnosis; Intraocular pressure; Syndrome; Glaucoma/diagnosis; Case reports

\section{ReFERÊNCIAS}

1. Tavares ADM, Holanda AGS, Tenório A, Dantas H, Araújo Z. Síndrome de Posner-Schlossman: relato de caso. Rev Bras Oftalmol. 2000;59(12):851-5.

2. Sellem E. Attitudes pratiques. Quand l'hypertension oculaire est unilatérale. J Fr Ophtalmol. 2006;29(Spec 2):52-6.
3. Barton K. Glaucoma secundário. In: Spalton DJ, Hitchings RA, Hunter PA. Atlas de oftalmologia clínica. 3a ed. São Paulo: Elsevier; 2006.

4. Kanski JJ. Oftalmologia clínica: uma abordagem sistemática. 6a ed. Rio de Janeiro: Elsevier; 2008.

5. Muccioli C, Finamor LP. Uveítes anteriores. In: Bicas HEA, Jorge AAH. Oftalmologia: fundamentos e aplicações. São Paulo: Tecmedd; 2007.

6. Pavan-Langston D, Galor A, Perez VL. Trato uveal: íris, corpo ciliar e coróide. In: Pavan-Langston D. Manual de diagnóstico e terapêutica ocular. 6a. ed. São Paulo: Tecmedd; 2007.

7. Jap A, Sivakumar M, Chee SP. Is Posner Schlossman syndrome benign? Ophthalmology. 2001;108(5):913-8. Comment in: Ophthalmology. 2002;109(3):409.

8. Dinakaran S, Kayarkar V. Trabeculectomy in the management of Posner-Schlossman syndrome. Ophthalmic Surg Lasers. 2002;33(4):321-2.

9. Yamamoto S, Pavan-Langston D, Tada R, Yamamoto R, Kinoshita S, Nishida K, et al. Possible role of herpes simplex virus in the origin of Posner-Schlossman syndrome. Am J Ophthalmol. 1995;119(6):796-8.

10. Wong M, Goldstein DA, Tessler HH. Presumed Fuchs heterochromic iridocyclitis and Posner-Schlossman syndrome: comparison of cytomegalovirus- positive and -negative eyes. Am J Ophthalmol. 2009;147(6):1106-7.

11. Choi CY, Kim MS, Kim JM, Park SH, Park KH, Hong C. Association between Helicobatcter pylori infection and Posner-Schlossman syndrome. Eye (Lond). 2000;24(1):64-9.

12. Hahn IH, Stillman MC. A case of glaucomatocyclitic crisis in the emergency department. Ann Emerg Med. 2006;47(2):167-9.

13. Leung DY, Rao SK, Lam DS. Pressure phosphene self-tonometry in a patient with Posner-Schlossman syndrome. Clin Experiment Ophthalmol. 2006;34(6):513-5.

14. Sociedade Brasileira de Glaucoma. $2^{\circ}$ Consenso Brasileiro de Glaucoma Primário de Ângulo Aberto. São Paulo: Planmark; 2005.

15. Pillai CT, Dua HS, Azuara-Blanco A, Sarhan AR. Evaluation of corneal endothelium and keratic precipitates by specular microscopy in anterior uveitis. $\mathrm{Br} \mathrm{J}$ Ophthalmol. 2000;84(12):1367-71.

16. Takahashi T, Ohtani S, Miyata K, Miyata N, Shirato S, Mochizuki M. A clinical elevation of uveitis-associated secondary glaucoma. Jpn J Ophthalmol. 2002;46(5):556-62.

17. Moorthy RS, Mermoud A, Baerveldt G, Minckler DS, Lee PP, Rao NA. Glaucoma associated with uveitis. Surv Ophthalmol. 1997;41(5):361-94. Review.

\section{Autores correspondentes:}

Paulo Ricardo Pereira de Oliveira

Rua Padre Chagas, n 66 - sala 603 - Moinhos de

Vento

CEP 90570-008 - Porto Alegre, (RS), Brasil

E-mail: pricardop.deoliveira@gmail.com

Tel: (51)3222-4806

César Gomes da Silveira

Residente CEPOA - RJ

R. Miguel Lemos, $n^{\circ} 10$ - 601- Copacabana

RJ, Brasil

E-mail: cesargsilveira@gmail.com

Tel: (21) 8138-9632 